\title{
Geomagnetic storms: historical perspective to modern view
}

\author{
Gurbax S. Lakhina ${ }^{1 *+}$ and Bruce T. Tsurutani ${ }^{2+}$
}

\begin{abstract}
The history of geomagnetism is more than 400 years old. Geomagnetic storms as we know them were discovered about 210 years ago. There has been keen interest in understanding Sun-Earth connection events, such as solar flares, CMEs, and concomitant magnetic storms in recent times. Magnetic storms are the most important component of space weather effects on Earth. We give an overview of the historical aspects of geomagnetic storms and the progress made during the past two centuries. Super magnetic storms can cause life-threatening power outages and satellite damage, communication failures and navigational problems. The data for such super magnetic storms that occurred in the last 50 years during the space era is sparce. Research on historical geomagnetic storms can help to create a database for intense and super magnetic storms. New knowledge of interplanetary and solar causes of magnetic storms gained from spaceage observations will be used to review the super magnetic storm of September 1-2, 1859. We discuss the occurrence probability of such super magnetic storms, and the maximum possible intensity for the effects of a perfect ICME: extreme super magnetic storm, extreme magnetospheric compression, and extreme magnetospheric electric fields.
\end{abstract}

Keywords: Super magnetic storms, Coronal mass ejections, Space weather

\section{Introduction: historical perspective}

In 1600 A. D., William Gilbert published De Magnete proposing that the Earth acts as a great magnet (Gilbert 1600). This led to the birth of Geomagnetism, a new branch of science at that time, with a great potential for ship navigation. By the beginning of the eighteenth century, the first map of magnetic field declination was prepared by Edmund Halley. The phenomenon of a magnetic storm was discovered by Alexander von Humboldt. He undertook the task of recording the local magnetic declination in Berlin, every half hour from midnight to morning, for the period starting from May 1806 until June 1807. On the night of December 21, 1806, von Humboldt observed strong magnetic deflections for six consecutive hours and noted the presence of correlated northern lights (aurora) overhead. He found that when the aurora disappeared at dawn, so did the magnetic perturbations. From these

\footnotetext{
*Correspondence: gslakhina@gmail.com

${ }^{\dagger}$ Gurbax S. Lakhina and Bruce T. Tsurutani have contributed equally for this paper.

${ }^{1}$ Indian Institute of Geomagnetism, New Panvel (W), Navi Mumbai, India Full list of author information is available at the end of the article
}

observations, von Humboldt concluded that the magnetic disturbances on the ground and the auroras in the polar sky were related to the same phenomenon. He called this phenomenon the "Magnetische Ungewitter" or a magnetic storm (von Humboldt 1808). Much later on, the worldwide network of magnetic observatories, which von Humboldt helped to setup, confirmed that such "magnetic storms" were worldwide phenomena (Schröder 1997).

In the beginning of the nineteenth century, research on geomagnetic activity and solar activity, namely, sunspot observations, were being studied independently. From 1826, S. Heinrich Schwabe, an amateur German astronomer, started observations of sunspots. In 1843, he reported a $\sim 10$-year periodic variation of sunspots (Schwabe 1843). In 1851, Lamont reported a 10 -year periodicity in the daily variation of magnetic declination at the Munich Observatory, but he did not relate it to the sunspot cycle (Lamont 1867; Schröder 1997). Sabine from his extensive studies (Sabine 1851, 1852), was the first to discover that geomagnetic activity paralleled the then recently discovered sunspot cycle. This established a connection between geomagnetic activity and sunspots. 
While studying a big group of sunspots in the morning of September 1, 1859, Richard Carrington was taken by surprise when he noticed the sudden appearance of "two brilliant beads of blinding white light" over the sunspots. The beads intensified with time for a while and then their intensity diminished, and finally they disappeared (Carrington 1859). This was the first well-documented observation of a white light (visible) solar flare on record. The September 1, 1859 solar flare was also observed by R. Hodgson (1859). However, more recently, the 1859 flare came to be known as the Carrington flare. On the very next day, a severe geomagnetic storm was observed. This geomagnetic storm was recorded by the Kew observatory, and some other observatories worldwide, especially Colaba, Bombay. Carrington knew about and noted the occurrence of the magnetic storm but he avoided connecting it with the solar flare. He wrote "one swallow does not make a summer" (Carrington 1859). It took nearly a century of concerted efforts by the scientific community to gather sufficient statistics to make a convincing case for an association between large solar flares and severe magnetic storms (Hale 1931; Chapman and Bartels 1600; Newton 1943).

With the advent of space era, there has been a tremendous impetus to understand solar-terrestrial relationships, including geomagnetic storms and their solar and interplanetary causes. A new branch of space sciences, namely, Space Weather has recently emerged. The regime of space weather extends over a vast region of the heliosphere, including the Sun, interplanetary space, planetary magnetospheres, ionospheres, atmospheres, and the ground. Geomagnetic storms form the core component of space weather. According to the modern definition, a geomagnetic storm is characterized by a main phase during which the horizontal component of the Earth's lowlatitude magnetic fields are significantly depressed over a time span of one to a few hours followed by its recovery, which may extend over several days (Rostoker et al. 1997). The cause of the storm is the intensification of the ring current (10-300 keV magnetospheric electrons and ions) and its movement closer to the Earth, thus, producing a depression in the geomagnetic field $\mathrm{H}$-component. The cause of the recovery phase is the decay of the ring current (loss of the energetic particles) due to charge exchange, Coulomb collisions, wave-particle interactions, and ring current energetic particle convection out of the magnetopause. The intensity of a geomagnetic storm is measured by the disturbance storm time (Dst) index or by the SYM-H index which is a measure of the symmetric ring current intensity (Iyemori 1990). Dst is an hourly index expressing the intensity of the ring current. The SYM-H index is the same as the Dst index-but computed at a higher time resolution of $1 \mathrm{~min}$ instead of $1 \mathrm{~h}$ used for Dst.
Super magnetic storms (SMSs) ( with Dst $<-500 \mathrm{nT}$ ) are relatively rare. In the space age (since 1958), only one true SMS has occurred. It occurred on 13-14 March 1989 and had an intensity of Dst $=-589 \mathrm{nT}$ (SYM-H $=-710$ $\mathrm{nT}$ ). Intense ionospheric currents during the SMS caused the Canadian Hydro-Quebec system to fail (Allen et al. 1989; Bolduc 2002). On November 20, 2003 there was a magnetic storm with Dst $\sim-490 \mathrm{nT}$ which almost reached the SMS level. These two are the only possible SMS events during the space age.

However, before this, a regularly maintained magnetic observatory network has been in existence for the past $\sim 175$ years. Research on historical geomagnetic storms can help to create an excellent database for magnetic storms of super intensities (Lakhina et al. 2005).

In this review, we first summarize the knowledge gained during the space era about the solar and interplanetary drivers of geomagnetic storms. Then, we discuss the case history of the super magnetic storm of September 1-2, 1859 (the Carrington event). This is followed with the discussion of the maximum possible intensity for a geomagnetic storm, the occurrence probability of super magnetic storms, and the conclusions.

\section{Review: modern view}

\section{Solar and interplanetary drivers of geomagnetic storms}

The immediate manifestations of solar (flare) events on the Earth are due to flare photons. The X-rays, extreme ultra violet (EUV) and ultraviolet (UV) emissions from solar flares are the first phenomenon to cause dayside ionization of the Earth's atmosphere (Mitra 1974; Tsurutani et al. 2005). The photons take only $\sim 8 \mathrm{~min}$ to travel from the Sun to the Earth. Somewhat later, the flare energetic particles and particles accelerated at the interplanetary coronal mass ejection (ICME) shocks, travel through space at nearly the speed of light and arrive at Earth (Tsurutani and Lin 1985; Cane et al. 1986; Kallenrode 2003). However, because the particles have to travel along the Parker magnetic field lines, their distance of travel is much greater than $1 \mathrm{AU}$, and thus they are delayed from the photons. The most energetic particles arrive in $10 \mathrm{~s}$ of min, but the greatest fluxes arrive in $\sim \mathrm{h}$. These energetic particles have access to the Earth's polar regions, causing ionization in those regions and worldwide radio blackouts (Tsurutani et al. 2009). The particles with sufficient intensities can cause satellite damage and radiation hazards for man in space as well (National Research Council report 2008; Royal Academy of Engineering report 2013). A much greater delayed effect is the ICME which can take a fraction of a day (Vaisberg and Zastenker 1976; Tsurutani et al. 2009) to days (Gonzalez and Tsurutani 1987; Gonzalez et al. 1989; Feynman and Gabriel 2000; Mannucci et al. 2005) to reach the Earth. With conditions 
of southwardly directed IMFs, the ICME and its sheath can cause geomagnetic storms and substorms (Rostoker and Falthammar 1967; Tsurutani and Meng 1972; Gonzalez et al. 1989; Echer and Gonzalez 2008a; Echer et al. 2008b). SMSs can cause increased drag of low Earthorbiting (LEO) satellites due to increased heating of the auroral zone atmosphere (Lei et al. 2008; Thayer et al. 2008) and uplift of the near-equatorial ionosphere (Mannucci et al. 2005; Tsurutani et al. 2012).

There is no one-to-one relationship between the occurrence of solar flares and CMEs or strong association between the strengths of the flares and the speed and magnetic intensities of the ICMEs. Intense $(-250$ $\mathrm{nT}<$ Dst $<-100 \mathrm{nT})$ ICME-related magnetic storms not associated with solar flares have been reported earlier by Tsurutani et al. (1988), Tang et al. (1989) and Tang and Tsurutani (1990), and recently by Kamide and Kusano (2015). It has also been shown that magnetic storms with intensities Dst $<-250 \mathrm{nT}$ are only caused by the magnetic cloud (MC) portions of ICMEs and not their upstream sheaths (Tsurutani et al. 1992; Echer and Gonzalez 2008a). CIRs do not cause such intense storms either (Tsurutani et al. 1995a, 1995b). However, for the super magnetic storms (Dst $<-500 \mathrm{nT}$ ) which we consider in this paper, large solar flares (energies $\sim 10^{24}$ to $10^{25} \mathrm{~J}$ ) always occur together with CME releases (Burlaga et al. 1981; Klein and Burlaga 1982). This is because magnetic reconnection at the Sun is responsible for both phenomena at these intense levels (Shibata et al. 1995; Magara et al. 1995; Benz 2008; Chen 2011; Shibata and Magara 2011).

CMEs may have speeds up to $3000 \mathrm{~km} \mathrm{~s}^{-1}$ near the Sun (Yashiro et al. 2004; Schrijver et al. 2012). If such an interplanetary CME (ICME) with intense southward interplanetary magnetic fields collide with the Earth's magnetosphere, it will cause an SMS (Tsurutani et al. 1992; Echer and Gonzalez 2008a). It is believed that the Carrington magnetic storm, the most intense magnetic storm (Dst $=-1760 \mathrm{nT}$ ) in recorded history, was caused by an ICME that collided with the Earth on September 1-2, 1859 (Tsurutani et al. 2003; Lakhina et al. 2012). The Carrington storm caused auroras to be visible down to $\pm 23^{\circ}$ magnetic latitude, at Hawaii and Santiago, Chile (Kimball 1960). It was presumably the auroral electrojet which came down to middle latitudes that induced currents at ground level over the United States and Europe, which in turn caused electrical shocks and fires by electrical arcing from telegraph wires (Loomis 1861; Tsurutani et al. 2015).

Modern society is becoming ever increasingly dependent on space technology for daily routine functions, such as communication, ship and satellite navigation, data transmission, global surveillance of resources, and atmospheric weather. However, if a Carrington-type storm (or a greater intensity one) were to occur now, it could cause much more damage to society than in 1859 when the telegraph was the latest technology of the time. Therefore, it is crucial to have knowledge about the occurrence of extreme events, and their causes in order to assess their possible impacts on society (Tsurutani et al. 2003; Cliver and Svalgaard 2004; Vasyliunas 2011; Lakhina et al. 2012; Hapgood 2012; Riley 2012; Cliver and Dietrich 2013; Cid et al. 2014).

\section{Case history: super magnetic storm of September 1-2, 1859}

The September 01, 1859 solar flare was reported together by Carrington (1859) and Hodgson (1859) in the Monthly Notices of the Royal Astronomical Society. The Carrington paper was the first thorough reportage of a white light (visible) solar flare. The solar flare was followed by a magnetic storm on September 1-2, 1859 at the Earth. The time delay between the flare time and the magnetic storm was $\sim 17 \mathrm{~h}$ and $40 \mathrm{~min}$ (Carrington 1859). This storm is the biggest magnetic storm in the recorded history. Tsurutani et al. (2003) reduced the Colaba Observatory (Mumbai, India) ground magnetometer data of September 1-3, 1859 and presented it to the scientific public. The auroral reports based on newspapers and personal correspondences with Sydney Chapman (Kimball 1960; Loomis 1861), and recently obtained (space age) knowledge of interplanetary causes of intense storms were applied to determine the probable causes of this super magnetic storm event. Here, we will briefly review the main characteristics of this storm (Tsurutani et al. 2003; Lakhina et al. 2005, 2012).

Figure 1 shows the deduced horizontal component magnetogram of September 1-2, 1859 from the Colaba Observatory recordings. The sudden impulse $\left(\mathrm{SI}^{+}\right)$preceding the storm had an intensity of $\sim+120 \mathrm{nT}$. The maximum $\mathrm{H}$-component depression during the storm main phase was $\Delta \mathrm{H} \approx-1600 \mathrm{nT}$. The duration of the main phase of the storm (corresponding to the presumed plasma injection) was $\sim 1.5 \mathrm{~h}$.

The Earth's plasmapause location during the storm main phase was estimated to be at $\mathrm{L}=1.3$ deduced from the auroral observational information. This information was used to determine the magnetospheric convection electric field, $E_{c} \sim 20 \mathrm{mV} \mathrm{m}^{-1}$. If one assumes a $10 \%$ magnetic reconnection efficiency (Gonzalez et al. 1989), one gets an interplanetary solar wind electric field of $E \sim 200 \mathrm{mV} \mathrm{m}^{-1}$.

The average shock transit speed of $V_{\text {shock }}=2380 \mathrm{~km}$ $\mathrm{s}^{-1}$ is readily deduced from the knowledge that the transit time of the ICME from the Sun to the Earth was $\sim 17 \mathrm{~h}$ and $40 \mathrm{~min}$. Then, using the empirical relationships between $V_{\text {sw }}$ (the solar wind speed at $1 \mathrm{AU}$ ) and 


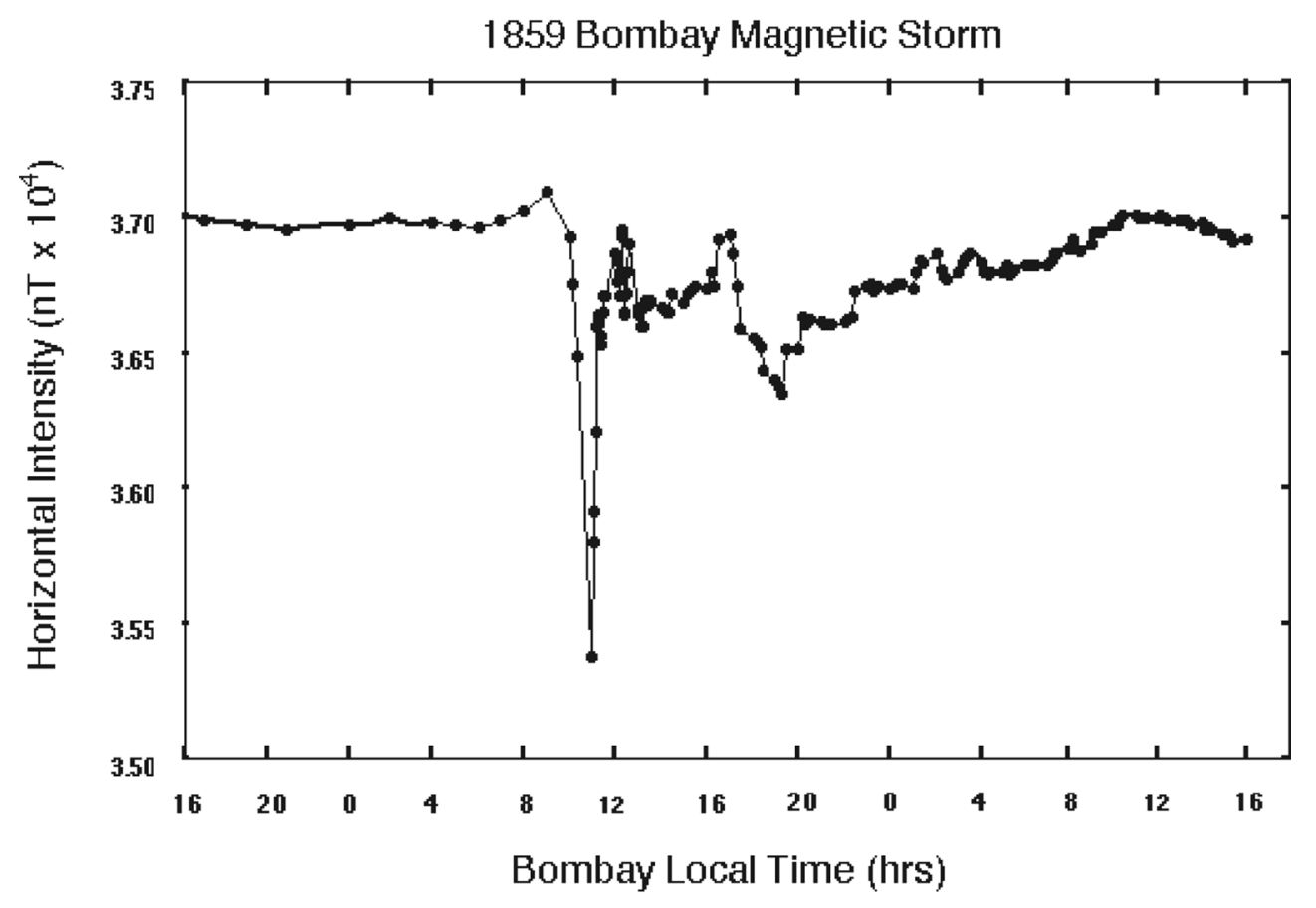

Sept. 1

Sept. 2

Sept. 3

Fig. 1 The Colaba (Bombay) magnetogram for the September 1-2, 1859 magnetic storm. From Tsurutani et al. (2003)

shock transit speed (Cliver et al. 1990), and the magnetic field $B$ of the ejecta at $1 \mathrm{AU}$ (Gonzalez et al. 1998), we get a solar wind speed $V_{\mathrm{sw}} \sim 1850 \mathrm{~km} \mathrm{~s}^{-1}$ and the magnetic cloud magnetic field magnitude $B \sim 90 \mathrm{nT}$ at $1 \mathrm{AU}$. The maximum possible electric field for this extremely fast ICME was calculated to be $E \sim 160 \mathrm{mV}$ $\mathrm{m}^{-1}$. This value compares well with the above estimate based on auroral location and reconnection efficiency $\left(E \sim 200 \mathrm{mV} \mathrm{m}^{-1}\right)$.

The empirical relation for the evolution of the ring current given by Burton et al. (1975) along with the ring current decay time of $1.5 \mathrm{~h}$ is used to estimate the peak intensity for this SMS event, giving Dst $=-1760 \mathrm{nT}$, a value consistent with the Colaba local measurement of $\Delta \mathrm{H}=-1600 \mathrm{nT}$.

It was concluded by Tsurutani et al. (2003) that a likely mechanism for the Carrington storm was intense $B_{\mathrm{s}}$ (southward) magnetic fields within a magnetic cloud (MC). The $\mathrm{MC}$ is one part of a CME, with coronal loops and a filament being the other two parts (Illing and Hundhausen 1986). The second and third depressions in Dst in Fig. 1 were probably caused by the new ring current injections from the successive ICMEs near the end of the fast recovery phase of the main storm, thus prolonging the overall "recovery" of the complex storm (Lakhina et al. 2012).
More recently, another possible scenario for the interplanetary cause of the Carrington storm has arisen. $\mathrm{Li}$ et al. (2006) performed computer simulations which suggested that a high density plasma plug could reproduce such a short time-scale storm event with a very fast recovery after the main phase of the storm. The authors did not identify the nature of this plasma at the time of their article. What could this "plasma plug" be? Kozyra et al. (2013) have found that high plasma density solar filaments (the most sunward part of CMEs) play prominent roles in extreme ICME events. Perhaps with more detailed research and simulations we will eventually know the answer of whether this hypothesis or another one can explain the 1859 storm in detail. An ICME sheath hypothesis can be ruled out because the magnetic field magnitudes within sheaths are far too weak to create SMS events (Echer and Gonzalez 2008a; Tsurutani et al. 1992; Kennel et al. 1985).

Is the very fast recovery after the main phase of the $\mathrm{H}$-component at Colaba for the Carrington storm unique? Cid et al. (2014) have shown that a less-thanone-hour recovery after the main phase is not uncommon in intense magnetic storms. They studied individual magnetic records of storm events of April 16, 1938 in Niemegk (NGK), March 14, 1989 in Borok (BOX), and October 29, 2003 in Tihany (THY), and discovered 
that these three events had magnetograms very similar in profile (but not magnitude) to the September 1-2, 1859 Carrington event recorded at Colaba, and all had very fast recovery after the main phases (see their Fig. 4). Cid et al. (2015) have analyzed the Carringtonlike event on October 29, 2003 at Tihany in detail. Based on their results of the October 29, 2003 event at Tihany, they have offered a re-interpretation of the 1859 event. According to the authors, the large drop in $\mathrm{H}$ recorded at Colaba during the Carrington storm could be caused by field-aligned currents, and not by the ring current. Whereas their hypothesis that field-aligned current caused the sharp decrease in H-component at Tihany for the October 29, 2003 event seems to be plausible, it should be noted that Tihany is a midlatitude $\left(\sim 46^{\circ}\right)$ station potentially vulnerable to strong magnetic signatures caused by ionospheric currents. The Cid et al. (2015) reinterpretation that field-aligned currents are the main cause for the 1859 Carrrington storm does not appear to be convincing as the Colaba Observatory is a near-equatorial $\left(\sim 10^{\circ}\right)$ station, located away from the equatorial electrojet influence and far away from severe storm-time auroral ionospheric current influences (Tsurutani et al. 2005).

It should be noted that the issue of the possibility of ionospheric currents causing the Colaba magnetic signature has already been addressed in the exchange between Akasofu and Kamide (2005) and Tsurutani et al. (2005). The former authors (Akasofu and Kamide 2005) stated "Magnetic changes of similar characteristics were, in fact, observed during some of the most intense magnetic storms, e.g., 11 February 1958 at Kakioka, Japan, and 15-16 July 1959 at Hermanus, South Africa. For example, Kakioka recorded an impulsive change of $-1000 \mathrm{nT}$, lasting $1 \mathrm{~h}$ during the former storm, versus $-500 \mathrm{nT}$ in the minimum Dst value." Tsurutani et al. (2005) responded: "It should be noted that Kakioka, Japan $\left(27^{\circ}\right.$ magnetic latitude) and Hermanus, South Africa ( $-34^{\circ}$ magnetic latitude), are located at middle latitudes and are potentially vulnerable to strong magnetic signatures caused by ionospheric currents. They would be useful for studying moderate-intensity magnetic storms but not extreme events such as the one in 1859."

Earlier, Cid et al. (2013) have found that a hyperbolic function, rather than the usual exponential function, can reproduce the recovery phase of the largest magnetic storms listed in Table 1 of Tsurutani et al. (2003). The hyperbolic function suggests that the losses of energy in the magnetosphere might be proportional to the square of the energy content and not to the energy content itself as implied by the exponential function decay. One possible physical mechanism for the rapid loss of ring current could be convection across the dayside magnetopause.

\section{Maximum possible intensity for a geomagnetic storm}

Although the Carrington storm is the most intense storm in recorded history, many of its associated properties are not the most extreme. That is because the relationship between flare energy, flare particle energy, CME speed, magnetic storm intensity, etc., are only loosely related. For example, the August 4, 1972 ICME was faster than that of 1859 ICME, with the highest ICME shock transit speed of $2850 \mathrm{~km} \mathrm{~s}^{-1}$ on record (Vaisberg and Zastenker 1976). The ICME transit time from the Sun to the Earth was $14.6 \mathrm{~h}$. Similarly, the ICME associated with the solar flare of October 28, $2003 \mathrm{had}$ an average speed of $\sim 2000$ $\mathrm{km} \mathrm{s}^{-1}$ and a transit time of $\sim 19 \mathrm{~h}$ (Mannucci et al. 2005; Skoug et al. 2004), but it could produce only an intense magnetic storm with Dst $=-358 \mathrm{nT}$. It is clear that the ICME that caused the Carrington storm was not unique in terms of speed. As pointed out earlier, it is the southward component of the magnetic fields, and not just the energy of the solar flare and speed of the ejecta, which control the strength of the geomagnetic storm. Therefore, fast ICMEs with strong southward magnetic fields in the magnetic cloud regions do have the potential to produce SMSs with intensities comparable or higher than the Carrington storms.

It is interesting to point out an event of a very fast CME with an initial speed of $2500 \mathrm{~km} \mathrm{~s}^{-1}$, but directed away from the Earth, as observed by STEREO-A on July 23, 2012. At $1 \mathrm{AU}$, the magnetic cloud of this ICME had an average transit speed of $1910 \mathrm{~km} \mathrm{~s}^{-1}$ and a peak magnetic field strength of $109 \mathrm{nT}$. This event has renewed interest in the study of extreme geomagnetic storms due to their potential impact on space- and ground-based technologies (Baker et al. 2013; Russell et al. 2013; Ngwira et al. 2013a, b). Had this powerful interplanetary event been Earthward directed, it would have produced a super intense magnetic storm with Dst $=-1182 \mathrm{nT}$ (Baker et al. 2013; Russell et al. 2013; Ngwira et al. 2013a, b). It has been suggested that strong magnetic cloud magnetic field was created by an interaction of two CMEs, where a shock driven by the second CME overtook the first CME from behind before the direct compression of the second CME occurred (Liu et al. 2014). This could have been the most intense magnetic storm of the space era, and could have caused severe hazardous space weather effects at Earth.

It is important to know the maximum possible effects a CME can cause so that damage to space and ground technologies can be protected. This problem has been analyzed recently by Tsurutani and Lakhina (2014). They explored the effects of a possible extreme CME when it hits the magnetosphere (storm intensity, $\mathrm{SI}^{+}$, electric fields, etc.). We shall briefly describe highlights of their model. Tsurutani and Lakhina (2014) considered the 
extreme value of CME speeds to be $3000 \mathrm{~km} \mathrm{~s}^{-1}$ near the Sun (Yashiro et al. 2004; Schrijver et al. 2012). The CME will be decelerated due to drag interaction with the high density slow solar wind in its propagation path. Solar active regions (ARs) generate multiple CME releases (and multiple flares) (Tsurutani et al. 2008, 2014) and the associated ICMEs tend to create a low interplanetary drag environment by "cleaning out" the upstream solar wind plasma. Under such a situation, Tsurutani and Lakhina (2014) took a $10 \%$ decrease as a maximum drag of the ICME, or a speed $\sim 2700 \mathrm{~km} \mathrm{~s}^{-1}$ at Earth. They then estimated the maximum values of the interplanetary shock strength, magnetospheric compression, the magnetospheric and ground electric and magnetic field pulses, and the magnetic storm intensity.

From the Rankine-Hugoniot conservation conditions (Tsurutani and Lin 1985), a shock speed $V_{\mathrm{s}}=3480 \mathrm{~km}$ $\mathrm{s}^{-1}$ relative to the spacecraft frame was obtained for the case of an upstream slow solar wind having the speed of $350 \mathrm{~km} \mathrm{~s}^{-1}$ and a proton number density of $5 \times 10^{6} \mathrm{~m}^{-3}$ and a downstream density value of $20 \times 10^{6} \mathrm{~m}^{-3}$ [a possible maximum jump of $\sim 4$ times as theoretically predicted by Kennel et al. (1985)]. Such a fast ICME shock can transit the $1 \mathrm{AU}$ distance from the Sun to the Earth in $\sim 12.0 \mathrm{~h}$. The Alfvén and magnetosonic Mach numbers of this shock were found to be $\sim 63$ and $\sim 45$, respectively. The ram pressure downstream of the ICME shock during impingement on the magnetosphere will push the magnetopause inwards from its quiet time position of $\sim 11.9$ $R_{\mathrm{e}}$ to a new subsolar position at $\sim 5.0 R_{\mathrm{e}}$ from the center of the Earth, where a $R_{\mathrm{e}}$ is an Earth radius $(6371 \mathrm{~km})$. The resulting sudden impulse $\mathrm{SI}^{+}$amplitude was estimated to be $\Delta \mathrm{H}$ of $\sim 234 \mathrm{nT}$ which exceeds the $\mathrm{SI}^{+}$amplitude of $202 \mathrm{nT} \mathrm{SI}^{+}$recorded at Kakioka, Japan on March 24, 1991 (Araki et al. 1997). The passage of the shock through the magnetosphere produced fast time variations in the magnetic field, of the order of $\mathrm{d} B / \mathrm{dt} \sim 30 \mathrm{nT} \mathrm{s}^{-1}$. This could cause a maximum magnetospheric electric field of the order of $1.9 \mathrm{~V} \mathrm{~m}^{-1}$. This is a remarkable result in view of the fact that a new radiation belt composed of $\sim 15 \mathrm{MeV}$ electrons was created low in the magnetosphere when an interplanetary shock hit the Earth's magnetosphere on March 24, 1991 (Blake et al. 1992; Li et al. 1993). The electric field amplitude for this event was estimated by Wygant et al. (1993) to be $\sim 300 \mathrm{mV} \mathrm{m}^{-1}$. Since the above hypothesized maximum SMS event will have an electric field 6 times the intensity of the 1991 case, it could produce a much stronger new radiation belt.

Tsurutani and Lakhina (2014) estimated the magnetic cloud field strength of $\sim 127 \mathrm{nT}$ from the empirical relationship between the speed and magnetic field strength of the ICME at 1 AU (Gonzalez et al. 1998). Assuming that the MC magnetic field is directed entirely southward, the interplanetary electric field strength of $\sim 340 \mathrm{mV} \mathrm{m}^{-1}$ was deduced. This is nearly twice the estimated value for the Carrington storm (Tsurutani et al. 2003). Since the magnetic storm intensity has been found to scale approximately linearly with the interplanetary electric field amplitude (Burton et al. 1975; Echer et al. 2008b), the maximum intensity of the magnetic storm could be twice the Carrington storm intensity, i.e., Dst -3500 nT. However, there is a maximum possible Dst limit of -2500 nT derived by Vasyliunas (2011) from an analysis based on plasma beta arguments. Thus, the maximum intensity of the magnetic storm associated with this extreme event will possibly be limited to Dst $\geq-2500$ $\mathrm{nT}$. This is an interesting prediction, as none of the SMSs, either past or present, has been close to this limit.

\section{Super magnetic storms and their Dst profiles}

Super magnetic storms are caused by solar ejecta (due only to CMEs as far as we know) having unusually intense southward magnetic fields, and high solar wind speeds near the Earth. Sheaths in general cannot cause storms of intensities at the SMS level, but can cause lesser intensity major storms. Dst profiles of different SMSs are, however, found to be dissimilar because of the different nature of the ICMEs causing them.

From Fig. 1, it is clear that the Carrington storm (Dst $=-1760 \mathrm{nT}$ ) was a single-step magnetic storm probably caused by an ICME magnetic cloud having intense southward magnetic fields (Tsurutani et al. 2003). The SMS has a short main phase of only an hour and a half.

In contrast, the March 13-14, 1989 storm, the only SMS event that occurred in the space age (Allen et al. 1989), was quite complex and had a long and complex main phase. Unfortunately, there was no interplanetary spacecraft/data upstream of the Earth's magnetosphere during this event. All that is available for analyses are the ground magnetograms. Figure 2 shows the SYM-H plot for this event. The maximum SYM-H value was $\sim-710 \mathrm{nT}$. The corresponding peak Dst value was $-589 \mathrm{nT}$. The storm main phase extends from $\sim 02 \mathrm{UT}$ 13 March to $\sim 01$ UT 14 March, with an interval of $\sim 23 \mathrm{~h}$.

There are two clear $\mathrm{SI}^{+}$events, one at the beginning of the event at $\sim 2$ UT 13 March and a second event at $\sim 0830$ UT on the same day. The first had a magnitude of $\sim+40 \mathrm{nT}$, and the second $\sim+80 \mathrm{nT}$. There appears to be two more likely $\mathrm{SI}^{+}$events at $\sim 11 \mathrm{UT}$ and $16 \mathrm{UT}$ with magnitudes of $\sim+50$ and $+65 \mathrm{nT}$, respectively. All the $\mathrm{SI}^{+} \mathrm{S}$ were most likely caused by interplanetary shocks leading sheaths or sheath compressions. Six clear intervals of decreasing SYM-H can be identified. The first main phase of the storm from $\sim 0230$ UT to up until $\sim 09$ UT, which produced SYM-H $=-150 \mathrm{nT}$, was probably due to sheath 


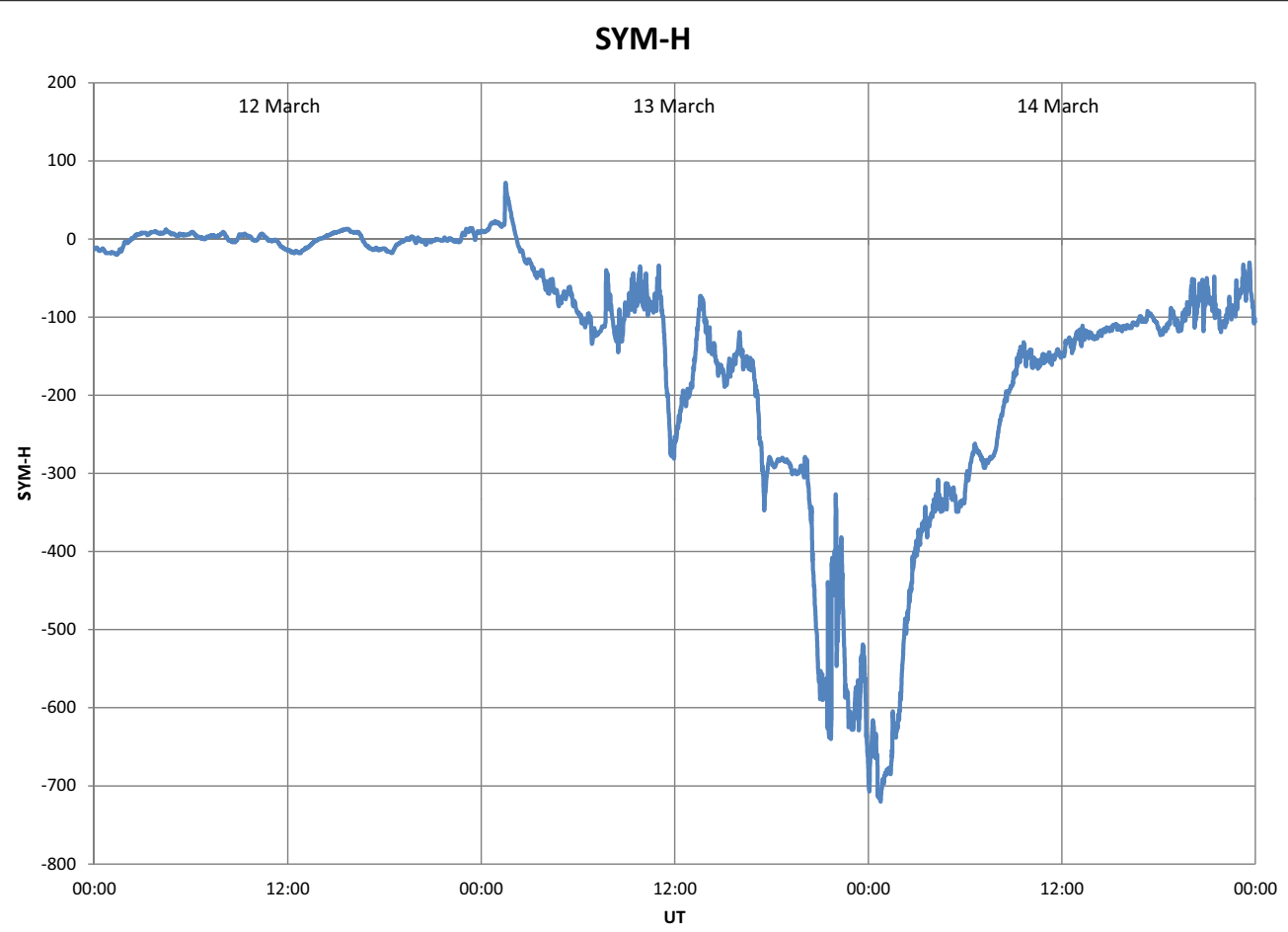

Fig. 2 The SYM-H profile of the March 13-14, 1989 super magnetic storm event

magnetic fields (Kamide et al. 1998). During the second main phase of the storm from $\sim 11$ UT-12 UT there is a sharp negative increase to SYM-H $\sim-260 \mathrm{nT}$. The sharp, smooth drop was most likely due to a MC southward $B_{\mathrm{z}}$ component. The third main phase of the storm has multiple sharp SYM-H negative excursions at 18 UT (SYM-H $\sim-350 \mathrm{nT}$ ), $21 \mathrm{UT}$ (SYM-H $\sim-580 \mathrm{nT}$ ), and $\sim 22 \mathrm{UT}$ (SYM-H -625 nT). The storm was clearly quite complex. These sharp SYM-H negative increases may have been generated by multiple MC southward magnetic fields. Near and at the peak of the multiple main phase storm, from 23 UT 13 March to 01 UT 14 March, the main phase becomes more monotonic ending at a peak SYM-H value of $-710 \mathrm{nT}$. This peak is followed by a recovery from $\sim 01$ to 06 UT 14 March. The SMS peak and recovery could have been generated by a south-north MC event.

The intense magnetic storms of October 29-30, 2003 have been suggested to have been caused by two fast ICMEs with speeds $\sim 2000 \mathrm{~km} \mathrm{~s}^{-1}$ (Mannucci et al. 2005; Alex et al. 2006). The first ICME and its upstream sheath caused a double storm with first depression in Dst -200 nT ( due to southward sheath magnetic field) occurring around 0900 UT on 29 October and the second depression due to southward MC field of Dst $=-350 \mathrm{nT}$ at $\sim 0125 \mathrm{UT}$ on 30 October. The duration of the storm main phase was $\sim 18 \mathrm{~h}$. Before this storm's recovery was complete, strong southward MC magnetic fields of the second ICME caused a new single step storm with a Dst $\sim-400 \mathrm{nT}$ at 2315 UT on October 30, 2003 (Mannucci et al. 2005). The duration of the main phase of this storm was $\sim 5 \mathrm{~h}$.

Lastly, we consider the Dst profile of the super magnetic storm of November 20, 2003 (Alex et al. 2006; Mannucci et al. 2008). This was a single-step storm (Dst $=-490 \mathrm{nT}$ ) caused by the intense southward magnetic field of the MC associated with an ICME traveling with a speed of about $1100 \mathrm{~km} \mathrm{~s}^{-1}$. The main phase lasted for about $8 \mathrm{~h}$. From the above arguments, we conclude that it is not just the amplitudes of the southward magnetic fields associated with ICMEs, but their duration too, play an important part in causing the the severity of the magnetic storms.

What will be the Dst profile of the possible extreme magnetic storm? It is not possible to predict it with certainty. However, we postulate that it will have a short single phase, somewhat like the Carrington storm, caused by the intense southward field of the MC associated with the extreme ICME. The sheath field, if southwardly oriented, could provide a small precursor storm to the larger MC-driven storm.

\section{Probability of super magnetic storms}

It is difficult to answer the questions: "how often can SMSs occur?" or "Can one assign probabilities to the 
occurrence of Carrington-type storms or SMSs?" To answer the first question, the ICME associated with one big flare (energy $\sim 10^{25} \mathrm{~J}$ ) per solar cycle (11 years) (Kane et al. 1995, 2005; Schrijver et al. 2012) has the potential for creating an SMS with an intensity similar to the 1859 storm. But, in reality, we know that the Carrington magnetic storm was the largest storm in the last 155 years (about 14 solar cycles). Thus, either no such big flare followed by an extreme ICME has occurred in the past, or if it did, it was not geoeffective. The answer to the question about the predictability of similar or greater intensity events requires knowledge of either the full understanding of the physical processes causing extreme ICMEs and magnetic storms or good empirical statistics of the tail of their distributions. Since we neither fully understand the physical processes, nor have good data of the tail distributions, in our opinion, it is not possible at this time to estimate their probabilities of occurrence with any reasonable accuracy. However, others have attempted to make some predictions, given caveats.

Tsubouchi and Omura (2007) have analyzed a 45-yearlong Dst dataset from 1957 to 2001 using the extreme value theory. Their model predicts an occurrence frequency of a March 1989 storm intensity (Dst $=-589$ nT)or greater as once in 60 years. They also calculate the occurrence rate of a Carrington-type magnetic storm (Dst $=-1760 \mathrm{nT}$ ) from their model, and they obtain one event every $\sim 40,000$ years.

Riley (2012) has predicted the probability of storms with Dst $<-850$ nT occurring within the next decade to be about $\sim 12 \%$ based on the assumption that the frequency of occurrence scales as an inverse power of the intensity of the event.

Love (2012) has estimated the most likely Poisson occurrence probability for another Carrington-type event in the next 10 years as 0.063 . Recently, Love et al. (2015) have applied the lognormal statistics to the Dst time series for the years 1957-2012, and predicted the maximum likelihood for a magnetic storm with intensity exceeding Dst $<-850 \mathrm{nT}$ to be about 1.13 times per century.

Kataoka (2013) has used a new statistical model of cumulative distribution functions based on the 89 year list of magnetic storms recorded at the Kakioka Magnetic Observatory to calculate the probability of extreme magnetic storms in solar cycle 24 . He estimates the probability of another Carrington-type storm occurring within the next decade to be $4-6 \%$.

Willis et al. (1997) have applied extreme value statistics to the first, second, and third largest geomagnetic storms per solar cycle for 14 solar cycles (1844-1993) using the daily $a a$ indices. They predict a $99 \%$ probability that there will not be a storm with $a a>550$ for the next 100 solar cycles.
Recent statistical analysis by Yermolaev et al. (2013) shows that occurrence frequency of Carrington-type storms cannot be higher than once every 500 years.

\section{Conclusions}

There has been tremendous progress in our understanding of the causes of intense magnetic storms during the past 50 years. We have applied the knowledge gained from the study of intense magnetic storms to identify the solar and interplanetary driver of the September 1-2, 1859 magnetic storm, the most intense magnetic storm in recorded history. A similar procedure can be adopted to study other SMSs where no interplanetary data is available. We have shown that the Dst or SYM-H profile of a SMS can possibly tell us about the nature of the interplanetary drivers that cause storms. Analysis of the SYM-H profile of the March 13-14, 1989 super magnetic storm was used to illustrate this point.

At this stage, it is not possible to make any accurate prediction of when or how often an extreme storm with similar or higher intensity than that of the September 1-2, 1859 event could occur. We have tried to answer here "what could the maximum intensity of a storm be?" by considering the case of an extreme CME having speed of $3000 \mathrm{~km} \mathrm{~s}^{-1}$ near the Sun, and a $10 \%$ decrease as a maximum ICME drag during its passage through the slow solar wind plasma from the Sun to 1 AU. Under ideal conditions, the upper limit of an ICME shock transit time from the Sun to the Earth is estimated to be $\sim 12.0 \mathrm{~h}$. The sheath impingement of the magnetosphere will push the magnetopause in to a distance of $5.0 R_{\mathrm{e}}$ from the center of the Earth, thus, exposing all geosynchronous spacecraft $\left(\mathrm{r}=6.6 R_{\mathrm{e}}\right)$ and outer magnetospheric spacecraft to the full brunt of solar wind and solar flare particles. The maximum interplanetary electric field at Earth is estimated to be $\sim 340 \mathrm{mV} \mathrm{m}^{-1}$. The magnetic storm intensity could reach at least the saturation value of Dst $=-2500$ nT predicted by Vasyliunas (2011).

However, we must emphasize that the above scenario would change if the energy of the interplanetary shock driven by the kinetic flow of the ICME goes primarily into shock acceleration of particles. This would lead to a deceleration of the ICME. If that happened then the extreme CME would be less geoeffective in creating a magnetic storm.

Furthermore, we have not taken into consideration the possibility of superflares ( flares exceeding the energy of $10^{25} \mathrm{~J}$ ) from the Sun. Schrijver et al. (2012) predict a probability of at most $10 \%$ for a solar flare exceeding energy of $10^{26} \mathrm{~J}$ in the next 30 years. Analyses of tree rings have identified large ${ }^{14} \mathrm{C}$ and ${ }^{10} \mathrm{Be}$ enhancement events in AD774-5 (Miyake et al. 2012; Usoskin and Kovaltsov 2012) and in AD992-3 (Miyake et al. 2013). If these events can 
be verified as true solar particle events (SPEs), then the particle energies will be $\sim 10^{26}-10^{28} \mathrm{~J}$. The occurrence rate of such large SPEs has been estimated as $10^{-4}-10^{-3}$ per year (Usoskin and Kovaltsov 2012). Recently, Maehara et al. (2012) have analyzed the Kepler space telescope data and reported that Sun-like stars can have superflares with energies up to $10^{28} \mathrm{~J}$, occurring once every 5000 years. On the other hand, the superflares with energies of $10^{26} \mathrm{~J}$ are found to have an average occurrence rate of about once in 500-600 years (Maehara et al. 2015). If such superflares were to occur on the Sun, the accompanying CMEs could be much more extreme than considered here, thus causing even stronger interplanetary and magnetospheric effects. This will have important implications for the Sun-Earth system. It should be mentioned that if superflares were associated with CME speeds higher than $3000 \mathrm{~km} \mathrm{~s}^{-1}$ at the Sun, then all of the interplanetary features (CME shock speed, energetic particle fluxes) and magnetospheric features (magnetospheric compression, $\mathrm{SI}^{+}$, magnetospheric electric fields, etc.) would scale accordingly.

\section{Authors' contributions}

This paper was presented as an invited talk in the special session ST21-32 during AOGS2015, Singapore, 02-07 August 2015 by GSL. Both the authors have contributed equally in writing the paper. Both authors have read and approved the final manuscript.

\section{Author details}

${ }^{1}$ Indian Institute of Geomagnetism, New Panvel (W), Navi Mumbai, India ${ }^{2}$ NASA Jet Propulsion Laboratory, Calfornia Institute of Technology, Pasadena, CA, USA.

\section{Acknowledgements}

Portions of this research were conducted at the Jet Propulsion Laboratory, California Institute of Technology under contract with NASA. GSL thanks the National Academy of Sciences, India, for support under the NASI-Senior Scientist Platinum Jubilee Fellowship. The authors would like to thank R. Hajra for help in the data analyses.

\section{Competing interests}

The authors declare that they have no competing interests.

Received: 8 October 2015 Accepted: 8 February 2016

Published online: 20 February 2016

\section{References}

Akasofu S-I, Kamide Y (2005) Comment on "The extreme magnetic storm of 1-2 September 1859" by B. T. Tsurutani, W. D. Gonzalez, G. S. Lakhina, and S. Alex. J Geophys Res 110:09226. doi:10.1029/2005JA011005

Alex S, Mukherjee S, Lakhina GS (2006) Geomagnetic signatures during the intense geomagnetic storms of 29 October and 20 November 2003. J Atmos Solar Terres Phys 68:769-780

Allen J, Sauer H, Frank L, Reiff P (1989) Effects of the March 1989 solar activity. EOS Trans AGU 70:1479

Araki T, Fujitani S, Emoto M, Yumoto K, Shiokawa K, Ichinose T, Luehr H, Orr D, Milling D, Singer H, Rostoker G, Tsunomura S, Yamada Y, Liu CF (1997) Anomalous sudden commencement on March 24, 1991. J Geophys Res 102:14075

Baker DN, Li X, Pulkkinen A, Ngwira CM, Mays ML, Galvin AB, Simunac KDC (2013) A major solar eruptive event in July 2012: defining extreme space weather scenarios. Space Weather 11:585-591
Benz AO (2008) Flare observations. Living Rev Solar Phys 5:1

Blake JB, Kolassinski WA, Fillius RA, Mullen EG (1992) Injection of electrons and protons with energies of tens of MeV into $L<3$ on March 24, 1991 Geophys Res Lett 19:821-824

Bolduc L (2002) GIC observations and studies in the Hydro-Quebec system. J Atmos Sol Terr Phys 64:1793

Burlaga LF, Sittler E, Mariani F, Schwenn R (1981) Magnetic loop behind an interplanetary shock: Voyager, Helios, and IMP 8 observations. J Geophys Res 86:6673-6684

Burton RK, McPherron RL, Russell CT (1975) Empirical relationship between interplanetary conditions and Dst. J Geophys Res 80:4204

Cane HV, McGuire RE, von Rosenvinge TT (1986) Two classes of solar energetic particle events associated with impulsive and long-duration soft X-ray flares. Ap J 301:448-459

Carrington RC (1859) Description of a singular appearance seen in the Sun on September 1, 1859. Mon Not R Astron Soc 20:13-15

Chapman S, Bartels J (1600) Geomagnetism. Oxford Univ. Press, New York, pp 328-337

Chen PF (2011) Coronal mass ejections: models and their observational basis. Living Rev Solar Phys 8:1

Cid C, Palacios J, Saiz E, Cerrato Y, Aguado J, Guerrero A (2013) Modeling the recovery phase of extreme geomagnetic storms. J Geophys Res 118:4352-4359

Cid C, Palacios J, Saiz E, Guerrero A, Cerrato Y (2014) On extreme geomagnetic storms. J Space Weather Space Clim 4:28. doi:10.1051/swsC/2014026

Cid C, Saiz E, Guerrero A, Palacios J, Cerrato Y (2015) A Carrington-like geomagnetic storm observed in the 21st century. J Space Weather Space Clim 5:16. doi:10.1051/swsc/2015017

Cliver EW, Dietrich WF (2013) The 1859 space weather event revisited: limits of extreme activity. J Space Weather Space Clim 3:31. doi:10.1051/ swsc/2013053

Cliver E, Feynman J, Garrett H (1990) An estimate of the maximum speed of the solar wind, 1938-1989. J Geophys Res 95:17103-17112

Cliver EW, Svalgaard L (2004) The 1859 solar-terrestrial disturbance and the current limits of extreme space weather activity. Solar Phys 224:407-422

Echer E, Gonzalez WD, Tsurutani BT (2008a) Interplanetary conditions leading to superintense geomagnetic storms (Dst $\leq-250 \mathrm{nT}$ ) during solar cycle 23. Geophys Res Lett 35:03-06. doi:10.1029/2007GL031755

Echer E, Gonzalez WD, Tsurutani BT, Gonzalez ALC (2008b) Interplanetary conditions causing geomagnetic storms (Dst $\leq-100 \mathrm{nT}$ ) during solar cycle 23 (1996-2006). J Geophys Res 113:05221. doi:10.1029/200 7JA012744

Feynman J, Gabriel SB (2000) On space weather consequences and predictions. J Geophys Res 105:10543

Gilbert W. De Magnete, Chiswick (1600) London

Gonzalez WD, Gonzalez ALC, Lago AD, Tsurutani BT, Arballo JK, Lakhina GS, Ho CM, Wu S-T (1998) Magnetic cloud field intensities and solar wind velocities. Geophys Res Lett 25:963-966

Gonzalez WD, Tsurutani BT (1987) Criteria of interplanetary parameters causing intense magnetic storms (Dst <100 nT). Planet Space Sci 35:1101-1109

Gonzalez WD, Tsurutani BT, Gonzalez ALC, Smith EJ, Tang F, Akasofu S-I (1989) Solar wind-magnetosphere coupling during intense magnetic storms (1978-1979). J Geophys Res 94:8835-8851

Hale GE (1931) The spectrohelioscope and its work. part III. Solar eruptions and their apparent terrestrial effects. Astrophys J 73:379-412

Hapgood MA (2012) Prepare for the coming space weather storm. Nature 484:311-313. doi:10.1038/484311a

Hodgson R (1859) On a curious appearance seen in the Sun. Mon Not R Astron Soc 20:15-16

Illing RM, Hundhausen JR (1986) Disruption of a streamer by an eruptive prominence and coronal mass ejection. J Geophys Res 91:10951-10960

lyemori T (1990) Storm-time magnetospheric currents inferred from midlatitude geomagnetic field variations. J Geomag Geoelec 42:1249-1265

Kallenrode M-B (2003) Current views on impulsive and gradual solar energetic particle events. J Phys G Nucl Part Phys 29:965. doi:10.1088/0954-3899/29/5/316

Kamide Y, Kusano K (2015) No major solar flares but the largest geomagnetic storm in the present solar cycle. Space Weather 13:365-367. doi:10.100 2/2015SW001213 
Kamide Y, Yokoyama N, Gonzalez W, Tsurutani B, Daglis I, Brekke A, Masuda S (1998) Two-step development of geomagnetic storms. J Geophys Res 103:6917-6921

Kane SR, Hurley K, McTiernan JM, Sommer M, Boer M, Niel M (1995) Energy release and dissipation during giant solar flares. Astrophys J 446:47-50

Kane SR, McTiernan JM, Hurley K (2005) Multi-spacecraft observations of the hard X-ray emission from the giant solar flare on 2003 November 4. Astron Astrophys 433:1133-1138. doi:10.1051/0004-6361:20041875

Kataoka R (2013) Probability of occurrence of extreme magnetic storms. Space Weather 11:214-218. doi:10.1002/swe.20044

Kennel CF, Edmiston JP, Hada T (1985) A quarter century of collisionless shock research. In: Stone RG, Tsurutani BT (eds) Collisionless Shocks in the Heliosphere: a Tutorial Review, Geophys Mono Ser vol. 34. AGU, Washington, pp. 1-36

Kimball DS (1960) A Study of the Aurora of 1859. Sci Rep 6, UAG-R109, Univ. of Alaska Fairbanks, Fairbanks

Klein LW, Burlaga LF (1982) Magnetic clouds at 1 AU. J Geophys Res 87:613

Kozyra JU IV, Escoubet WBM, Lepri CP, Liemohn MW, Gonzalez WD, Thomsen MW, Tsurutani BT (2013) Earth's collision with a solar filament on 21 January 2005: overview. J Geophys Res 118:5967-5978. doi:10.1002/ jgra.50567

Lakhina GS, Alex S, Tsurutani BT, Gonzalez WD (2005) Research on historical records of geomagnetic storms. In: Dere KP, Wang J, Yan Y (eds) Coronal and Stellar Mass Ejections: Proceedings of the 226th Symposium of the International Astronomical Union Held in Beijing, 13-17 Sep 2004, pp 3-15 (2005). Cambridge Univ. Press, Cambridge

Lakhina GS, Alex S, Tsurutani BT, Gonzalez WD (2012) Supermagnetic storms: hazard to society. In: Sharma AS, Bunde A, Dimri VP, Baker DN (eds) Extreme Events and Natural Hazards: The Complexity Perspective, Geophys. Mon. Ser. vol. 196. AGU, Washington, p. 267. doi:10.1029/2011GM001073

Lamont J (1867) Handbuch des Magnetismus Leipzig

Lei J, Thayer JP, Forbes JM, Wu Q, She C, Wan W, Wang W (2008) Ionosphere response to solar wind high-speed streams. Geophys Res Lett 35:19105. doi:10.1029/2008GL035208

Li XL, Roth I, Temerin M, Wygant JR, Hudson MK, Blake JB (1993) Simulation of the prompt energization and transport of radiation belt particles during the March 24, 1991 SSC. Geophys Res Lett 20:2423-2426

Li X, Temerin M, Tsurutani BT, Alex S (2006) Modelling of 1-2 September 1859 super magnetic storm. Adv Space Res 38:273-279

Liu YD, Luhmann JG, Kajdic P, Kilpua EK, Lugaz N et al (2014) Observations of an extreme storm in interplanetary space caused by successive coronal mass ejections. Nature Commun 5:3481

Loomis E (1861) On the great auroral exhibition of Aug 28th-Sept 4, 1859, and on auroras generally. Am J Sci 82:318

Love JJ (2012) Credible occurrence probabilities for extreme geophysical events: earthquakes, volcanic eruptions, magnetic storms. Geophys Res Lett 39:10301. doi:10.1029/2012GL051431

Love JJ, Rigler EJ, Pulkkinen A, Riley P (2015) On the lognormality of historical magnetic storm intensity statistics: implications for extreme-event probabilities. Geophys Res Lett 42:6544-6553. doi:10.1002/201 5GL064842

Maehara H, Shibayama T, Notsu Y, Notsu S, Honda S, Nogami D, Shibata K (2015) Statistical properties of superflares on solar-type stars based on 1-min cadence data. Earth, Planets and Space 67:59. doi:10.1186/ s40623-015-0217-z

Maehara H, Shibayama T, Notsu S, Notsu Y, Nagao T, Kusaba S, Honda S, Nogami D, Shibata K (2012) Superflares on solar-type stars. Nature Lett. 485:478-481. doi:10.1038/nature11063

Magara T, Shibata K, Yokoyama T (1995) Evolution of eruptive flares. I. plasmoid dynamics in eruptive flares. Astrophys J 487:437-446

Mannucci AJ, Tsurutani BT, Abdu MA, Gonzalez WD, Komjathy A, Echer E, lijima BA, Crowley G, Anderson D (2008) Superposed epoch analysis of the dayside ionospheric response to four intense geomagnetic storms. J Geophys Res 113:00-02. doi:10.1029/2007JA012732

Mannucci AJ, Tsurutani BT, lijima BA, Komjathy A, Saito A, Gonzalez WD, Guarnieri FL, Kozyra JU, Skoug R (2005) Dayside global ionospheric response to the major interplanetary events of October 29-30 2003 "Halloween storms". Geophys Res Lett 32:12. doi:10.1029/2004GL021467

Mitra AP (1974) lonospheric Effects of Solar Flares. Springer, New York
Miyake F, Masuda K, Nakamura T (2013) Another rapid event in the Carbon-14 content of tree rings. Nature Comm. 4:1748. doi:10.1038/ncomms2783

Miyake F, Nagaya K, Masuda K, Nakamura T (2012) A signature of cosmicray increase in AD 774-775 from tree rings in Japan. Nature Lett 486:240-242. doi:10.1038/nature11123

National Research Council Report (2008) Severe Space Weather EventsUnderstanding Societal and Economic Impacts. National Academies Press, Washington

Newton HW (1943) Solar flares and magnetic storms. Mon Not R Astron Soc 103:244-257

Ngwira CM, Pulkkinen A, Kuznetsova MM, Glocer A (2013b) Modeling extreme Carrington-type space weather events using three-dimensional global MHD simulations. J Geophys Res 119:4456-4474. doi:10.1002/201 3JA019661

Ngwira CM, Pulkkinen A, Mays ML, Kuznetsova MM, Galvin AB et al (2013a) Simulation of the 23 July 2012 extreme space weather event: what if this extremely rare cme was earth directed? Space Weather 11:671-679

Riley P (2012) On the probability of occurrence of extreme space weather events. Space Weather 10:02012. doi:10.1029/2011SW000734

Rostoker G, Falthammar C-G (1967) Relationship between changes in the interplanetary magnetic field and variations in the magnetic field at the Earth's surface. J Geophys Res 72:5853-5863

Rostoker G, Friedrich E, Dobbs M (1997) Physics of magnetic storms In: Tsurutani BT, Gonzalez WD, Kamide Y, Arballo JK (eds) Magnetic Storms, Geophys. Monogr. Ser. vol. 98. AGU, Washington pp. 149-160

Royal Academy of Engineering Report (2013) Extreme Space Weather Impacts on Engineered Systems and Infrastructure. Royal Academy of Engineering, London

Russell CT, Mewaldt RA, Luhmann JG, Mason GM, von Rosenvinge TT et al (2013) The very unusual interplanetary coronal mass ejection of 2012 July 23: a blast wave mediated by solar energetic particles. Astrophys J $770: 38$

Sabine $E$ (1851) On periodical laws discoverable in mean effects on the larger magnetic disturbances. Philos Trans R Soc London 141:103-129

Sabine $E$ (1852) On periodical laws discoverable in mean effects on the larger magnetic disturbances, ii. Philos Trans R Soc London 142:234-235

Schrijver CJ, Beer J, Baltensperger U, Cliver EW, Güdel M et al (2012) Estimating the frequency of extremely energetic solar events, based on solar, stellar, lunar, and terrestrial records. J Geophys Res 117:08103. doi:10.1029/ 2012JA017706

Schröder W (1997) Some aspects of the earlier history of solar terrestrial physics. Planet Space Sci 45:395-400

Schwabe SH (1843) Solar observations during 1843. Astron Nachr 20(495):234-235

Shibata K, Magara T (2011) Solar flares: magnetohydrodynamic processes. Living Rev Solar Phys 8:6

Shibata K, Masuda S, Shimojo M, Hara H, Yokoyama T, Tsuneta S, Kosugi T, Ogawara Y (1995) Hot plasma ejections associated with compact-loop solar flares. Astrophys J Lett 451:83

Skoug RM, Gosling JT, Steinberg JT, McComas DJ, Smith CW, Ness NF, Hu Q Burlaga LF (2004) Extremely high speed solar wind: 29-30 October 2003. J Geophys Res 109:09102. doi:10.1029/2004JA010494

Tang F, Tsurutani BT (1990) Reply. J Geophys Res 95:10721

Tang F, Tsurutani BT, Gonzalez WD, Akasofu SI, Smith EJ (1989) Solar sources of interplanetary southward $B_{z}$ events responsible for major magnetic storms (1978-1979). J Geophys Res 94:3535-3541

Thayer JP, Lei J, Forbes JM, Sutton EK, Nerem RS (2008) Thermospheric density oscillations due to periodic solar wind highspeed streams. J Geophys Res 113:06307. doi:10.1029/2008JA013190

Tsubouchi K, Omura Y (2007) Long-term occurrence probabilities of intense geomagnetic storm events. Space Weather 5:12003. doi:10.1029/200 7SW000329

Tsurutani BT et al (2005) The October 28, 2003 extreme EUV solar flare and resultant extreme ionospheric effects: Comparison to other Halloween events and the Bastille day event. Geophys Res Lett 32:03-09. doi:10.10 29/2004GL021475

Tsurutani BT, Echer E, Guarnieri FL, Kozyra JU (2008) CAWSES November 7-8, 2004, superstorm: complex solar and interplanetary features in the post-solar maximum phase. Geophys Res Lett 35:05-06. doi:10.1029/2 007GL031473 
Tsurutani BT, Echer E, Shibata K, Verkhoglyadova OP, Mannucci AJ, Gonzalez WD, Kozyra JU, Pätzold M (2014) The interplanetary causes of geomagnetic activity during the 7-17 March 2012 interval: a CAWSES ii overview. J Space Weather Space Clim 4:02. doi:10.1051/swsc/2013056

Tsurutani BT, Gonzalez WD, Gonzalez ALC, Guarnieri FL, Gopalswamy N, Grande M, Kamide Y, Kasahara Y, Lu G, Mann I, McPherron R, Soraas F, Vasyliunas V (1995b) Corotating solar wind streams and recurrent geomagnetic activity: A review. J Geophys Res 111:01-07. doi:10.1029/ 2005JA011273

Tsurutani BT, Gonzalez WD, Gonzalez ALC, Tang F, Arballo JK, Okada M (1995a) Interplanetary origin of geomagnetic activity in the declining phase of the solar cycle. J Geophys Res 100:21717-21733

Tsurutani BT, Gonzalez WD, Lakhina GS, Alex S (2003) The extreme magnetic storm of 1-2 September 1859. J Geophys Res 108:1268. doi:10.1029/2 002JA009504

Tsurutani BT, Gonzalez WD, Lakhina GS, Alex S (2005) Reply to comment by S.-I. Akasofu and Y. Kamide on "The extreme magnetic storm of 1-2 September 1859". J Geophys Res 110:09227. doi:10.1029/2005JA011121

Tsurutani BT, Gonzalez WD, Tang F, Akasofu SI, Smith EJ (1988) Origin of interplanetary southward magnetic field responsible for major magnetic storms near solar maximum (1978-1979). J Geophys Res 93:8519

Tsurutani BT, Gonzalez WD, Tang F, Lee YT (1992) Great magnetic storms. Geophys Res Lett 19:73

Tsurutani BT, Hajra R, Echer E, Gjerloev JW (2015) Extremely intense (SML $\leq-2500 \mathrm{nT}$ ) substorms: isolated events that are externally triggered? An Geo Comm 33:519-524. doi:10.5194/angeocom-33-519-2015

Tsurutani BT, Lakhina GS (2014) An extreme coronal mass ejection and consequences for the magnetosphere and Earth. Geophys Res 41:287-292. doi:10.1002/2013GL058825

Tsurutani BT, Lin RP (1985) Acceleration of $>47 \mathrm{keV}$ ions and $>2 \mathrm{keV}$ electrons by interplanetary shocks at 1 AU. J Geophys Res 90:1

Tsurutani BT, Meng C-I (1972) Interplanetary magnetic field variations and substorm activity. J Geophys Res 77:2964
Tsurutani BT, Verkhoglyadova OP, Mannucci AJ, Lakhina GS, Li G, Zank GP (2009) A brief review of "solar flare effects" on the ionosphere. Radio Sci 44:0-17

Tsurutani BT, Verkhoglyadova OP, Manucci AJ, Lakhina GS, Huba JD (2012) Extreme changes in the dayside ionosphere during a Carringtontype magnetic storm. J Space Weath Space Clim 2:05. doi:10.1051/ swsc/20122004

Usoskin IG, Kovaltsov GA (2012) Occurrence of extreme solar particle events: assessment from historical proxy data. Astrophys. J. 757:92. doi:10.1088/0004-637X/757/1/92

Vaisberg OL, Zastenker GN (1976) Solar wind and magneosheath observations at Earth during August 1972. Space Sci Rev 19:687

Vasyliunas VM (2011) The largest imaginable magnetic storm. J Atm Sol Terr Phys 73:1444. doi:10.1016/j.jastp.2010.05.012

von Humboldt A (1808) Die vollständigste aller bisherigen beobachtungen über den einfluss des nordlichts auf die magnetnadel. Ann Phys 29:425-429

Willis DM, Stevens PR, Crothers SR (1997) Statistics of the largest geomagnetic storms per solar cycle (1844-1993). Ann Geophys 15:719-728

Wygant J, Mozer F, Temerin M, Blake J, Maynard N, Singer H, Smiddy M (1993) Large amplitude electric and magnetic field signatures in the inner magnetosphere during injection of $15 \mathrm{MeV}$ electron drift echoes. Geophys Res Lett 21:1739-1742

Yashiro S, Gopalswamy N, Michalek G, Cyr OCS, Plunkett SP, Rich NB, Howard RA (2004) Acatalog of white light coronal mass ejections observed by the SOHO spacecraft. J Geophys Res 109:07105. doi:10.1029/200 3JA010282

Yermolaev YI, Lodkina IG, Nikolaeva NS, Yermolaev MY (2013) Occurrence rate of extreme magnetic storms. J Geophys Res 118:4760-4765. doi:10.1002/jgra.50467

\section{Submit your manuscript to a SpringerOpen ${ }^{\circ}$ journal and benefit from:}

- Convenient online submission

- Rigorous peer review

- Immediate publication on acceptance

- Open access: articles freely available online

- High visibility within the field

- Retaining the copyright to your article

Submit your next manuscript at $\boldsymbol{s p r i n g e r o p e n . c o m ~}$ 